\title{
Robust mode conversion in NV centers using exceptional points
}

\author{
A. Pick $\odot,{ }^{1,2, *}$ S. Silberstein, ${ }^{3}$ N. Moiseyev,,${ }^{1,4}$ and N. Bar-Gill ${ }^{3,5}$ \\ ${ }^{1}$ Faculty of Chemistry, Technion-Israel Institute of Technology, Haifa, Israel \\ ${ }^{2}$ Faculty of Electrical Engineering, Technion-Israel Institute of Technology, Haifa, Israel \\ ${ }^{3}$ Department of Physics, Hebrew University, Jerusalem 9190401, Israel \\ ${ }^{4}$ Faculty of Physics, Technion-Israel Institute of Technology, Haifa, Israel \\ ${ }^{5}$ Department of Applied Physics, Hebrew University, Jerusalem 9190401, Israel
}

(Received 3 May 2019; published 27 August 2019)

\begin{abstract}
We show that microwave-driven NV centers can function as robust mode switches by utilizing a special degeneracy called an exceptional point (EP). While previous theoretical and experimental work on EP-based mode switches applies only to pure states, we develop here a general theory for switching between mixed states, statistical ensembles of different pure states, resulting from the interaction with the environment. Our theory is general and applicable to all leading platforms for quantum information processing and quantum technologies. However, our numerical simulations use empirical parameters of NV centers. We provide guidelines for coping with the main challenges for experimental realization of this protocol: decoherence and mixed-state preparation.
\end{abstract}

DOI: 10.1103/PhysRevResearch.1.013015

A new class of adiabatic protocols enables robust mode conversion in open systems that possess a special degeneracy called an exceptional point (EP), where multiple modes of the system coalesce [1-3]. EP-based mode switches have intriguing physical properties, such as topological protection and nonreciprocity [4-8], which were demonstrated experimentally in optical waveguides and optomechanics [9-14] and theoretically proposed for several additional systems [15-18]. Realizing robust nonreciprocal mode switching in quantum systems has far-reaching consequences in quantum information processing and quantum control, as well as in quantum technology. Here, we show how to realize EP-based mode switches in atomic and atomlike systems. While previous work on EP-based mode switches applies only to pure states, the theoretical description of atomlike systems typically requires mixed states, statistical ensembles of different pure states, which arise due to interactions with the environment. To bridge this gap, we develop a theory of mode switching between mixed states. Our protocol applies, most generally, to three-level systems in the V configuration, and we perform numerical simulations using empirical parameters of nitrogenvacancy (NV) centers, defects in diamond with exceedingly long coherence lifetimes and established optical and microwave mechanisms for initialization, manipulation, and readout of their spin state [19-22]. Our theory enables exploring new phenomena (e.g., high-order EPs in low-dimensional systems) and presents a crucial step toward incorporating EPbased mode switches in quantum-information applications.

\footnotetext{
*pick.adi@gmail.com

Published by the American Physical Society under the terms of the Creative Commons Attribution 4.0 International license. Further distribution of this work must maintain attribution to the author(s) and the published article's title, journal citation, and DOI.
}

Robust mode switches are based on the adiabatic theorem, which describes the evolution of slowly varying closed systems. The theorem states that when preparing a system in a particular eigenmode, it remains in that mode during the evolution (given the conditions specified in Refs. [23,24]). Dynamic closed systems are described by Hermitian Hamiltonians that depend on a set of "control parameters." When changing the parameters slowly along closed loops in parameter space, the theorem implies that the initial and final states are the same (up to a phase [25-27]). The understanding that topological operations (i.e., executing closed control paths) may have outcomes that are robust against noise has led to important discoveries in multiple areas of physics [28-31].

However, most physical systems exchange energy or particles with their environment. Open systems can be described by effective non-Hermitian operators, and their adiabatic transport properties are drastically different from closed ones. First, unlike Hermitian operators, non-Hermitian operators may have EPs, where multiple eigenmodes have the same eigenvalue and eigenvector [1-3]. Near EPs, the eigenvalues are multivalued functions of the system's parameters and, consequently, when changing the control parameters along any closed loop that encircles an EP, the eigenmodes at the initial and final points may differ [32]. For example, when the EP is formed by the coalescence of two modes (hereafter called an EP2), the eigenmodes swap, i.e., $|1\rangle \rightarrow|2\rangle$ and $|2\rangle \rightarrow$ $|1\rangle$. Second, in non-Hermitian systems, the adiabatic theorem holds only for "least decaying states" [33-35], which are eigenmodes $\ell$ with eigenvalues $\lambda_{\ell}$ whose accumulated decay rate is positive:

$$
\Gamma_{\ell}(t) \equiv \int_{0}^{t} \operatorname{Re}\left[\lambda_{\ell}\left(t^{\prime}\right)-\lambda_{j}\left(t^{\prime}\right)\right] d t^{\prime}>0, \quad \forall j \neq \ell
$$

for all $t$ during the evolution (see Appendix A). When only two modes are involved, the accumulated decay rates $\Gamma_{1,2}(t)$ have opposite signs, which implies that while one of the states 
(a)

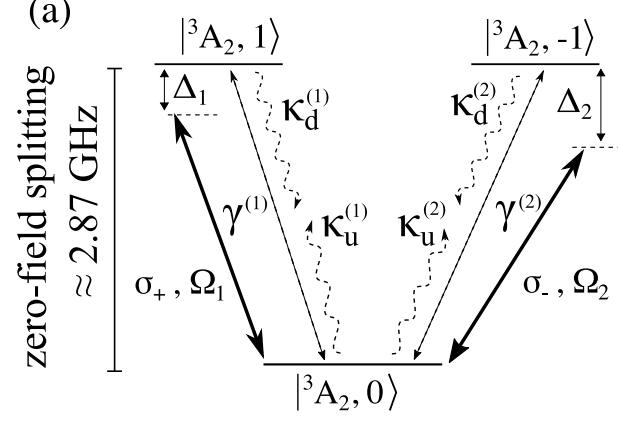

(b)

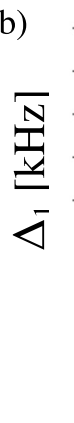

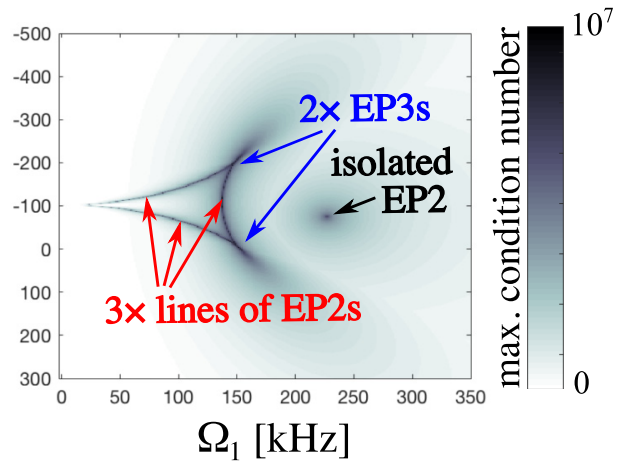

FIG. 1. (a) Ground states of the NV center. Two microwave fields drive transitions between the $\left|{ }^{3} A_{2}, 0\right\rangle$ and $\left|{ }^{3} A_{2}, \pm 1\right\rangle$ states, with Rabi frequencies $\Omega_{1,2}$, detunings $\Delta_{1,2}$, and left-/right-circular polarizations $\sigma_{ \pm}$. Interaction with the environment causes pure dephasing at a rate $\gamma^{(1,2)}$ (straight dashed arrows) and downward or upward transitions at rates $\kappa_{u}^{(1,2)}$ or $\kappa_{d}^{(1,2)}$, respectively (wiggly dashed arrows). The evolution of the system is governed by the Lindbladian operator $\hat{\mathcal{L}}$ [Eq. (3)]. (b) Exceptional points in the Lindbladian. A filled contour plot of the maximal condition number of the eigenvalues of $\hat{\mathcal{L}}$ [Eq. (7)]. We scan $\Delta_{1}$ and $\Omega_{1}$ while fixing all other parameters: $\Omega_{2}=400, \Delta_{2}=1400, \kappa_{u}^{(1,2)}=$ $\kappa_{d}^{(1,2)}=1, \gamma^{(1)}=900$, and $\gamma^{(2)}=1500$ (in $\mathrm{kHz}$ ). The condition number diverges when modes coalesce at exceptional points (EPs) (scale on the right).

can evolve adiabatically, the other one cannot. Therefore, when encircling an EP2 along a certain path, either $|1\rangle \rightarrow|2\rangle$ or $|2\rangle \rightarrow|1\rangle$ [4,5]. For any given loop, when reversing the direction of the path, the sign of the accumulated decay rate is reversed [since the order of integration limits in Eq. (1) is exchanged], and this is the source for "nonreciprocity" of EP-based switches [9,10].

The performance of the switch is robust since it is related to a topological property of the system's energy surfaces, the existence of an EP, which is a branch point in the system's energy surfaces $[9,10]$. In the adiabatic limit, the output of the switch depends only on whether or not the loop encircles an EP. Typically, small perturbations in the operational details only slightly distort the loop or move the location of the $\mathrm{EP}$, but do not lift the degeneracy. The possibility of creating nonreciprocal switches between pure states has attracted considerable attention [4-18] and, here, we generalize this concept for mixed states. Our protocol includes (i) finding an isolated EP in the eigenvalue spectrum of the NV center (Fig. 1), (ii) initializing the system in special superposition states (Fig. 2), and (iii) changing the control parameters in a loop around the EP (Fig. 3).

Figure 1(a) shows the electronic ground-state manifold of the negatively charged NV center (also called $\mathrm{NV}^{-}$). It consists of three spin-triplet states $\left|{ }^{3} A_{2}, m_{s}\right\rangle$, where $m_{s}=$ $0, \pm 1$ denotes the spin projection along the $\mathrm{NV}$ axis and $A_{2}$ marks the orbital symmetry [36,37]. In the absence of external magnetic fields, the energy levels of $\left|{ }^{3} A_{2}, 0\right\rangle$ and $\left|{ }^{3} A_{2}, \pm 1\right\rangle$ are split by $2.87 \mathrm{GHz}$. We introduce right- and left-circularly polarized transverse microwave fields to selectively drive the $\left|{ }^{3} A_{2}, 0\right\rangle \leftrightarrow\left|{ }^{3} A_{2}, \pm 1\right\rangle$ transitions $^{1}$ [39-41] (solid arrows). Using a semiclassical description [42] (where the electrons are treated quantum mechanically and the fields are treated classically) and employing the rotating-wave approximation [43] (which is valid when the driving fields are nearly resonant

\footnotetext{
${ }^{1}$ Alternatively, by applying a longitudinal magnetic field to lift the degeneracy of the $\left|{ }^{3} A_{2}, \pm 1\right\rangle$ states [38], one can excite these states selectively with a linearly polarized microwave field.
}

and relatively weak), the Hamiltonian of the driven NV center in the ground-state manifold is [43]

$$
\begin{aligned}
\hat{H}= & \Delta_{1}|1\rangle\left\langle 1\left|+\Delta_{2}\right|-1\right\rangle\langle-1|-\hbar \Omega_{1}(|1\rangle\langle 0|+| 0\rangle\langle 1|) \\
& -\hbar \Omega_{2}(|-1\rangle\langle 0|+| 0\rangle\langle-1|),
\end{aligned}
$$

where $\Omega_{i} \equiv \frac{\mathcal{E}_{i} \mu_{i}}{\hbar}$ denotes the Rabi frequency of each microwave field (with $\mathcal{E}_{i}$ the field amplitude, $\mu_{i}$ the transition dipole moment, and $i=1,2)$ and $\Delta_{i} \equiv E_{i}-E_{0}-\hbar \omega_{i}$ denotes the single-photon detuning, i.e., the frequency offset from each atomic transition (with $E_{0, i}$ the energies of states $|0, \pm 1\rangle$ and $\omega_{i}$ the microwave frequencies).

The electronic state of the system is described by a density matrix, whose evolution is governed by the Lindblad master equation $[43,44]$. In the Heisenberg picture, the Lindblad equation of motion for any observable $\hat{X}$ is

$$
\dot{\hat{X}} \equiv \hat{\mathcal{L}}[\hat{X}]=\frac{i}{\hbar}[\hat{H}, \hat{X}]+\sum_{j} \Gamma_{j}\left(2 \hat{L}_{j}^{\dagger} \hat{X} \hat{L}_{j}-\left\{\hat{L}_{j}^{\dagger} \hat{L}_{j}, \hat{X}\right\}\right)
$$

The first term represents Hamiltonian evolution and the remaining terms describe incoherent processes due to the interaction with the environment. We consider two types of processes [44,45]: (i) pure dephasing at a rate $\gamma^{(1,2)}$ (straight dashed arrows) and (ii) downward or upward jumps at rates $\kappa_{d}^{(1,2)}$ or $\kappa_{u}^{(1,2)}$, respectively (wiggly dashed arrows). At thermal equilibrium, the ratio of upward and downward transitions is given by the Boltzmann factor $\kappa_{u}^{(1,2)} / \kappa_{d}^{(1,2)}=$ $\exp \left(\hbar \omega_{1,2} / k_{B} T\right)$, where $k_{B} T$ is the thermal energy [45]. We assume that the system is at room temperature $(\approx 300 \mathrm{~K})$, where upward and downward transition rates are almost equal. ${ }^{2}$ The incoherent processes are described by [46]

$$
\begin{array}{ll}
\text { pure dephasing: } & \hat{L}_{1,2}=| \pm 1\rangle\langle \pm 1|-| 0\rangle\langle 0| \\
\text { up/down jumps: } & \hat{L}_{3,4}=| \pm 1\rangle\left\langle 0\left|, \quad \hat{L}_{5,6}=\right| 0\right\rangle\langle \pm 1|
\end{array}
$$

\footnotetext{
${ }^{2}$ It is possible to enhance the rate of downward transitions using green light [36]. This flexibility allows searching a wider parameter range, as will be explored in future work.
} 
(a)

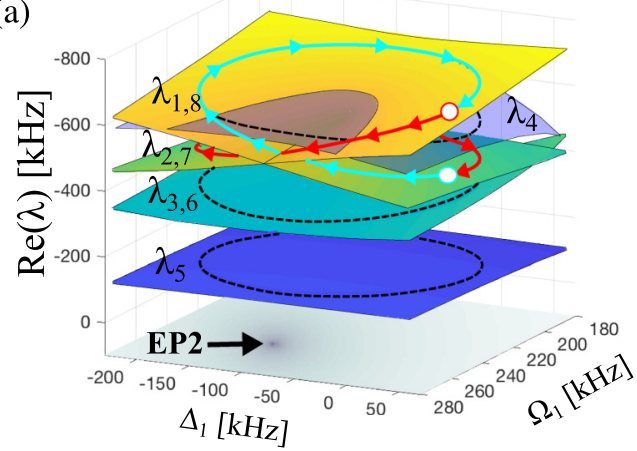

(b)

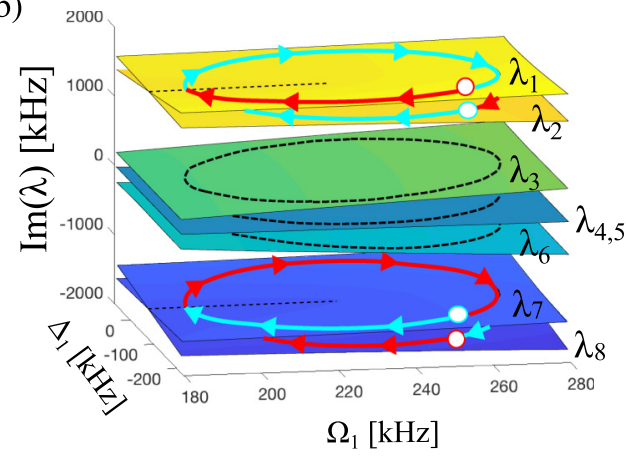

FIG. 2. Real and imaginary parts of the eigenvalues of the Lindbladian operator near the isolated EP. We scan $\Delta_{1}$ and $\Omega_{1}$ while holding all other parameters fixed (given in Fig. 1). In this regime, the Lindbladian has four simple eigenvalues $\left(\lambda_{3,4,5,6}\right)$ and two complex-conjugate pairs $\left(\lambda_{1,2}\right.$ and $\left.\lambda_{7,8}\right)$ that coalesce at the EP2. When changing the parameters in a loop around the EP2 (details in Appendix D), the simple eigenvalues return to the starting point (dashed lines) while the remaining eigenstates swap (arrowed lines), i.e., schematically, $|1\rangle \leftrightarrow|2\rangle$ and $|7\rangle \leftrightarrow|8\rangle$. The red-rimmed points (associated with $\left.\lambda_{1,8}\right)$ are transferred into cyan-rimmed points $\left(\lambda_{2,7}\right)$ and vice versa.

In order to find EPs in the eigenvalue spectrum of $\hat{\mathcal{L}}$, we rewrite the superoperator equation [Eq. (3)] in a form that is more convenient for theoretical investigation [46,47]. We introduce a basis of traceless orthogonal matrices, which spans the space of density matrices [48-50]. Specifically, we choose the eight Gell-Mann matrices $\left(\hat{\sigma}_{1}, \ldots, \hat{\sigma}_{8}\right.$, defined in Appendix B), which generalize Pauli matrices for three-level systems [51]. By applying Eq. (3) to each Gell-Mann matrix $\left(\hat{\sigma}_{i}\right)$ and taking the expectation value of the resulting equation, we obtain

$$
\dot{\vec{S}}=\hat{M}\left(\vec{S}-\vec{S}_{\mathrm{eq}}\right) .
$$

Here, $\vec{S}$ is an eight-dimensional vector, whose entries are $S_{i}=\operatorname{Tr}\left[\hat{\rho} \cdot \hat{\sigma}_{i}\right]$ and $\hat{\rho}$ is the density matrix. The real parts of the eigenvalues of $\hat{M}$ are the relaxation rates of the eigenmodes, while $\vec{S}_{\text {eq }}$ is the steady state. Explicit expressions for $\hat{M}$ and $\vec{S}_{\text {eq }}$ are given in Appendix B [Eqs. (B2) and (B4), respectively]. We consider here room temperatures, where $\vec{S}_{\mathrm{eq}} \approx 0$, since the rates of incoherent upward and downward transitions are equal. Left and right eigenvectors and eigenvalues of $\hat{M}$ satisfy

$$
\hat{M} \vec{S}_{i}^{R}=\lambda_{i} \vec{S}_{i}^{R}, \quad \hat{M}^{T} \vec{S}_{i}^{L}=\lambda_{i} \vec{S}_{i}^{L},
$$

where the superscript $T$ denotes matrix transposition. The matrix $\hat{M}$ is non-Hermitian and, hence, can have EPs. At an EP, the left and right eigenvectors of the degenerate eigenmode are orthogonal [52] (i.e., $\vec{S}_{i}^{L} \cdot \vec{S}_{i}^{R}=0$ ). Therefore, the condition number, defined as the secant of the angle between left and right eigenvectors [53],

$$
N\left(\lambda_{i}\right) \equiv \frac{1}{\left|\cos \left(\theta_{i}\right)\right|}=\frac{\left|\vec{S}_{i}^{L}\right|\left|\vec{S}_{i}^{R}\right|}{\left|\vec{S}_{i}^{L} \cdot \vec{S}_{i}^{R}\right|},
$$

diverges at the EP.

Figure 1(b) reveals the location of EPs in the eigenvalue spectrum of $\hat{\mathcal{L}}$. We scan the parameters of the right-circularly polarized field $\left(\Omega_{1}\right.$ and $\left.\Delta_{1}\right)$ while holding all other parameters fixed (see caption). At each point in parameter space, we compute the condition numbers of the eigenvalues of $\hat{M}$ and, then, plot the maximal condition number attained. The dark regions in the figure mark the location of the EPs. We determine the order of the degeneracy by plotting the eigenvectors at selected points along the dark lines. We find three lines of EP2s, which intersect at two points of EP3s (similar to [45,54]) and an isolated $\mathrm{EP} 2$ at $\left(\Delta_{1}^{\mathrm{EP}}, \Omega_{1}^{\mathrm{EP}}\right) \approx(-80,225) \mathrm{kHz}$. The same system can be used for finding fourth- and fifth-order EPs, as we show in Appendix C.

Next, we demonstrate swapping of the instantaneous eigenvalues along loops that encircle the isolated EP2. Figure 2 shows surfaces of the real and imaginary parts of the eigenvalues of $\hat{M}$ [Eq. (B2)] as a function of $\Delta_{1}$ and $\Omega_{1}$. In the shown parameter regime, $\hat{M}$ has four simple eigenvalues $\left(\lambda_{3,4,5,6}\right)$ and two complex-conjugate pairs of eigenvalues $\left(\lambda_{1,2}\right.$ and $\left.\lambda_{7,8}\right)$ that coalesce at the isolated EP2. We choose a loop that encircles the EP2 and, then, compute the eigenvalues at each point along the path. (The path details are given in Appendix D). As expected, the simple eigenvalues return to the starting point after the loop (dashed lines), while the remaining eigenstates swap (arrowed lines). That is, $|1\rangle \leftrightarrow|2\rangle$ and $|7\rangle \leftrightarrow|8\rangle$; the red-rimmed points (associated with $\lambda_{1,8}$ ) are transferred into cyan-rimmed points (associated with $\lambda_{2,7}$ ) after the cycle and vice versa.

Having shown that the eigenmodes of $\hat{M}$ swap when encircling the EP2, let us adopt a more intuitive picture and transform the Gell-Mann vectors into density matrices. For three-level systems, the transformation is [50]

$$
\hat{\rho}=\frac{1}{3}\left(\hat{\mathbb{1}}+\sqrt{3} \sum_{i=1}^{8} \hat{\sigma}_{i} S_{i}\right),
$$

where $\hat{\sigma}_{i}$ are the Gell-Mann matrices (see Appendix B). In order to conserve the probabilistic interpretation of $\hat{\rho}, \vec{S}$ must be real [48-50]. Consequently, complex eigenmodes (such as $\vec{S}_{1,2}^{R}$ ) cannot be used as inputs for the switch and, instead, we initialize it in symmetric superpositions of complex-conjugate vectors, i.e., $\vec{S}_{\text {in }}^{(1)} \propto \vec{S}_{1}^{R}+\vec{S}_{8}^{R}$ and $\vec{S}_{\text {in }}^{(2)} \propto \vec{S}_{2}^{R}+\vec{S}_{7}^{R}$. Positivity of $\hat{\rho}$ implies that the length of $\vec{S}$ needs to be smaller than a critical value [50]. To satisfy this condition, we normalize $\vec{S}_{\text {in }}^{(1)}$ and $\vec{S}_{\text {in }}^{(2)}$ accordingly. Last, we use (8) to transform the Bloch vectors $\vec{S}_{\text {in }}^{(1,2)}$ into density matrices $\hat{\rho}_{\text {in }}^{(1,2)}$.

Next, we simulate the evolution of the system. We initialize the system in either $\hat{\rho}_{\text {in }}^{(1)}$ or $\hat{\rho}_{\text {in }}^{(2)}$ and solve Eq. (5) via the 

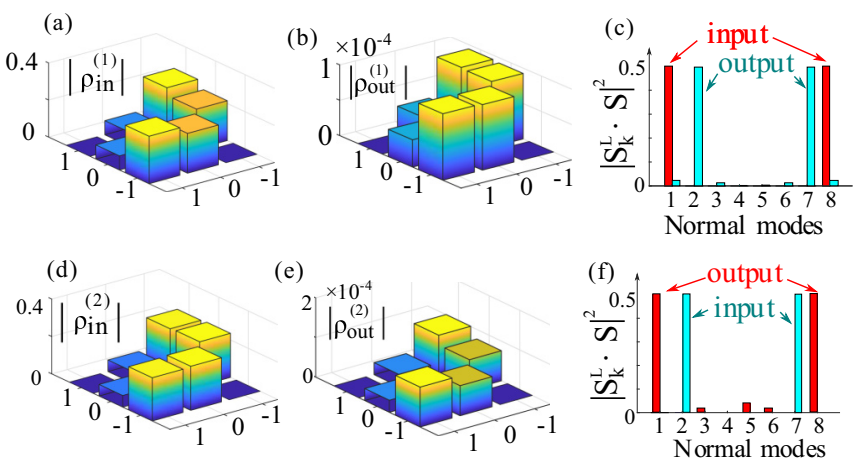

FIG. 3. Nonreciprocal mode switches in NV centers. We initialize the system in either $\hat{\rho}_{\text {in }}^{(1)}$ or $\hat{\rho}_{\text {in }}^{(2)}$ and let it evolve as the parameters are varied along the path from 2 . Panels (a), (b), (d), and (e) show the moduli of the off-diagonal density-matrix elements at the input and output. (Diagonal entries are set to zero for clarity.) (a), (b) The initial state $\hat{\rho}_{\text {in }}^{(1)}$ is adiabatically transported into $\hat{\rho}_{\text {out }}^{(1)} \propto \hat{\rho}_{\text {in }}^{(2)}$ after a clockwise loop around the EP. (d), (e) The initial state $\hat{\rho}_{\text {in }}^{(2)}$ is adiabatically transported into $\hat{\rho}_{\text {out }}^{(2)} \propto \hat{\rho}_{\text {in }}^{(1)}$ after a counterclockwise loop. (c), (f) Normalized projections of the initial and final states on the basis states at $t=0$.

standard Runge-Kutta method [53]. Figure 3 summarizes our main result: The initial state $\hat{\rho}_{\text {in }}^{(1)}$ is adiabatically transported into $\hat{\rho}_{\text {in }}^{(2)}$ when the parameters are varied in a clockwise manner around the EP [Figs. 3(a) and 3(b)]. This happens because $\hat{\rho}_{\text {in }}^{(1)}$ is the least-decaying eigenstate for a clockwise loop, which guarantees that the probability for quantum jumps into different eigenstates is negligible. Conversely, $\hat{\rho}_{\text {in }}^{(2)}$ is adiabatically transported into $\hat{\rho}_{\text {in }}^{(1)}$ only after a counterclockwise loop around the EP [Figs. 3(d) and 3(e)]. More details about the dynamics are given in Appendix D (Fig. 5). Figures 3(c) and 3(f) show the projections of the initial and final Gell-Mann vectors on the eigenvectors at the beginning of the loop, reaffirming that $\vec{S}_{\text {in }}^{(1)} \rightarrow \vec{S}_{\text {in }}^{(2)}$ after a clockwise loop while $\vec{S}_{\text {in }}^{(2)} \rightarrow \vec{S}_{\text {in }}^{(1)}$ after a counterclockwise loop.

In order for the system to evolve adiabatically, the sweep rates of $\Delta_{1}$ and $\Omega_{1}$ need to be small compared to the "energy gap" [55] (i.e., the distance between the complex eigenvalues). It implies that the cycle needs to be long compared to the dephasing time (e.g., we chose $\left.T=15 / \gamma^{(1)}\right)$. Consequently, the final states $\vec{S}_{\text {out }}^{(1,2)}$ approach zero [since $\vec{S}_{\text {eq }} \approx 0$ ]. From Eq. (8), one learns that when $|\vec{S}| \ll 1$, $\hat{\rho}$ is nearly diagonal, and it is hard to distinguish the final states from diagonal matrices. For visual clarity, in Fig. 3, we set the diagonal elements of $\hat{\rho}$ to zero, and show only the moduli of the off-diagonal elements. Since these terms are very small $\left[O\left(10^{-4}\right)\right]$, it is challenging to distinguish between the final states. Possible approaches for fighting decoherence are discussed in the concluding paragraph.

Another challenge arises from the fact that the input states $\hat{\rho}_{\text {in }}^{(1,2)}$ are mixed. Experimentally, preparing pure states is a standard procedure [36] but the preparation of mixed states is more challenging. One approach for overcoming this problem is to prepare the system in pure states in the vicinity of $\hat{\rho}_{\text {in }}^{(1,2)}$. The problem of finding the nearest pure state to a given density matrix is equivalent to finding the best rankone approximation for that matrix, which can be solved by exploiting the singular value decomposition [56,57]. Unfortunately, when applying this algorithm to $\hat{\rho}_{\text {in }}^{(1,2)}$, one obtains pure states that have significant population in undesired states, ${ }^{3}$ which deteriorate the performance of the switch. Alternatively, one could use quantum control to find protocols for preparing arbitrary mixed states [58-61]. However, experimental implementation of such protocols may be very difficult. These directions will be explored in future work.

To summarize, we presented a protocol for achieving robust mode conversion with NV centers by using EPs. Our work generalizes the existing theory of EP-based switches in two aspects: (1) by including mixed states, which are necessary for describing most existing platforms for quantum information processing, and (2) by treating multilevel systems, generalizing previous work that focused on two-level systems. We find that EP-based mode switches in Lindbladian systems require at least three electronic levels, and that one could force high-order EPs by carefully tuning several control parameters (see Fig. 4). Our analysis raises two challenges for experimental demonstration of this protocol: mixed-state preparation and decoherence. The former challenge is technical, and several ways for addressing it are mentioned above. The latter, fighting decoherence, is more fundamental. Quite generally, there is an incompatibility between adiabaticity (which implies slow evolution) and maintaining coherence (which requires, in turn, fast evolution) [35]. Adiabaticity requires that the parameter sweep rate should be smaller than the energy gap [23] which, in our case, is on the order of the decoherence rate. Unfortunately, it implies that whenever the evolution period is long enough to enable adiabatic evolution, the signal degrades substantially. This shortcoming can be remedied by using modified protocols. For example, one could introduce additional lasers that actively restore the lost coherence during the evolution. Such "cycling transitions" exist in NV centers, and require cold-temperature conditions [38]. Another possibility is to use the notion of PT symmetry, which implies that under some appropriate conditions, a PTsymmetric non-Hermitian system evolves without dissipation $[62,63]$. PT-symmetric evolution was recently observed in NV centers [64], and this approach can be extended to design a PT-symmetric mode switch. Such protocols are expected to significantly improve the performance of the switch, and will be addressed in future work.

The authors would like to thank R. Kosloff, S. Rahav, A. Mailybaev, Y. Kurman, S. Wolf, and I. Meirzada for helpful discussions. A.P. is supported by an Aly Kaufman Fellowship at the Technion and by The Center for Absorption in Science, Ministry of Immigrant Absorption, State of Israel. N.M. acknowledges the financial support of I-Core: The Israeli Excellence Center "Circle of Light," and of the Israel Science Foundation (Grant No. 1530/15). N.B. acknowledges support from the European Union (ERC StG, MetaboliQs), the CIFAR

\footnotetext{
${ }^{3}$ The pure states have significant population in $\vec{S}_{5}^{R}$, which is the steady state since $\left|\operatorname{Re}\left[\lambda_{5}\right]\right|<\left|\operatorname{Re}\left[\lambda_{j}\right]\right|$ for all $j \neq 5$ [see Fig. 2(a)]. Since the cycle is much longer than the coherence lifetime, the final state is almost precisely $\vec{S}_{5}^{R}$, and the swapping of the partial populations of $\vec{S}_{\text {in }}^{(1)}$ and $\vec{S}_{\text {in }}^{(2)}$ becomes unmeasurable.
} 

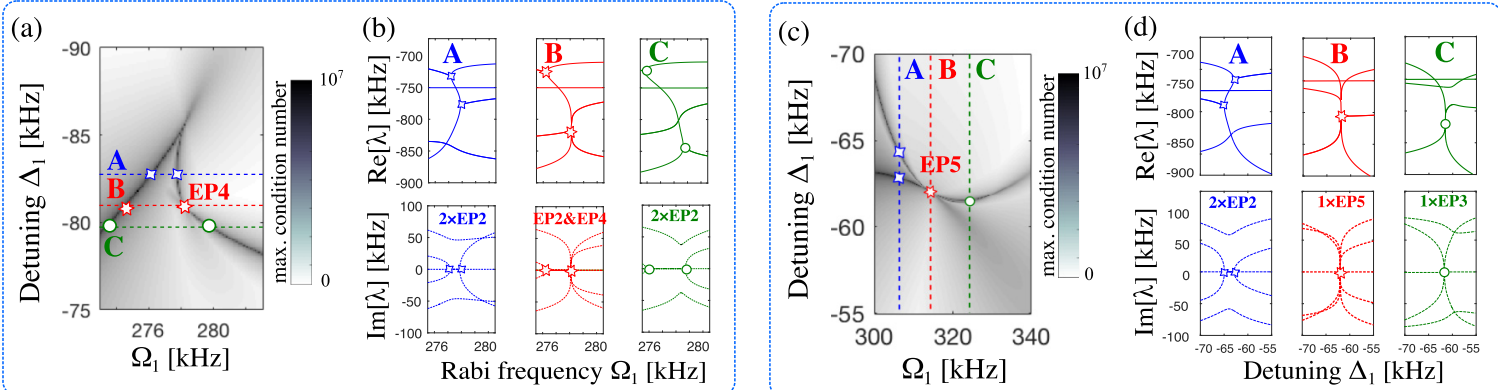

FIG. 4. Higher-order EPs, found by searching the four-dimensional parameter space spanned by the two Rabi frequencies $\Omega_{1,2}$ and frequency offsets $\Delta_{1,2}$ [see Fig. 1(a)]. (a) A fourth-order degeneracy is found at $\Delta_{1}=-80.8, \Omega_{1}=278.2, \Delta_{2}=44.3, \Omega_{2}=-445$ (in units of kHz). (b) The plots show real and imaginary parts of the eigenvalues (top and bottom plots) as a function of $\Omega_{1}$, at three values of $\Delta_{1}$, above (A) at (B) and below the EP (C). The plots demonstrate that two pairs of second-order EPs (EP2s) merge into an EP4. (c) A fifth-order degeneracy is found at $\Delta_{1}=-62, \Omega_{1}=314, \Delta_{2}=58, \Omega_{2}=436$. (d) Similar to (b), the plots show the real and imaginary parts of the eigenvalues along the three cuts (labeled A-C) in (c). The plots demonstrate that two pairs of EP2s merge with an EP3 to form the fifth-order EP.

Azrieli Global Scholars program, the Ministry of Science and Technology, Israel, and the Israel Science Foundation (Grant No. 750/14).

\section{APPENDIX A: ADIABATIC THEOREM FOR NON-HERMITIAN SYSTEMS}

In this Appendix, we sketch the proof of the adiabatic theorem for non-Hermitian systems following [33]. Let us investigate the conditions for adiabatic evolution of the normal modes of a non-Hermitian time-dependent operator $\hat{M}(\varepsilon t)$ (with $\varepsilon>0$ ). We introduce a new variable, $s=\varepsilon t$, and consider the limit of $\varepsilon \rightarrow 0$ while $s$ is held fixed and finite. By invoking the normal-mode expansion of $\hat{M}(s)$, one can write

$$
\hat{M}(s)=\sum_{j=1}^{n} \lambda_{j}(s)\left|\psi_{j}^{R}(s)\right\rangle\left\langle\psi_{j}^{L}(s)\right|,
$$

where the time-dependent right and left eigenvectors and corresponding eigenvalues are defined in Eq. (6) in the main text. The normal modes satisfy the biorthogonality condition $\left\langle\psi_{i}^{L}(s) \mid \psi_{j}^{R}(s)\right\rangle=\delta_{i j}$. Let $|\psi(s)\rangle$ be a solution of the differential equation

$$
\varepsilon \frac{\partial}{\partial s}|\psi(s)\rangle=\hat{M}(s)|\psi(s)\rangle .
$$

Substituting the following ansatz

$$
|\psi(s)\rangle=\sum_{j} a_{j}(s) \exp \left(-\frac{1}{\varepsilon} \int_{s_{0}}^{s} \lambda_{j}(u) d u\right)
$$

into Eq. (A2) and using the biorthogonality condition, one obtains

$$
\begin{aligned}
\partial_{s} a_{\ell}(s) & +\left\langle\psi_{\ell}^{L}(s)\left|\partial_{s} \psi_{\ell}^{R}(s)\right| a_{\ell}(s)\right. \\
= & -\sum_{j \neq \ell}\left\langle\psi_{\ell}^{L}(s)\left|\partial_{s} \psi_{j}^{R}(s)\right| a_{j}(s)\right. \\
& \times \exp \left(-\frac{1}{\varepsilon} \int_{s_{0}}^{s}\left[\lambda_{j}(u)-\lambda_{\ell}(u)\right] d u\right) .
\end{aligned}
$$

From Eq. (A4), one can easily read the conditions for adiabatic evolution. For Hermitian systems [where $\hat{M}(s)=$
$\left.\hat{M}^{\dagger}(s)\right]$, the eigenvalues $\left[\lambda_{j}(s)\right]$ are real and the right-hand side of (A4) contains only rapidly oscillating terms, which average to zero in the limit of $\varepsilon \rightarrow 0$. Conversely, when $\hat{M}(s)$ is non-Hermitian, the right-hand side of (A4) can be neglected only when

$$
\Gamma_{\ell j}(s)=\int_{s_{0}}^{s} \operatorname{Re}\left[\lambda_{j}(u)-\lambda_{\ell}(u)\right] d u>0
$$

for all $j \neq \ell$. To summarize, the condition for adiabatic evolution comes from requiring that the probability to jump from state $\ell$ to $j$ is small. In contrast to Hermitian systems, where the probability for quantum jumps oscillates in time, in nonHermitian systems, it also contains exponentially growing or decaying factors, and can be small only if $\Gamma_{\ell j}(t)>0$ for all $j \neq \ell$.

\section{APPENDIX B: VECTORIZING THE LINDBLAD MASTER EQUATION}

In this Appendix we derive Eq. (5) from the main text and present explicit expressions for the dynamical matrix $\hat{M}$ and the steady-state Gell-Mann vector $\vec{S}_{\text {eq }}$. A similar formulation was introduced in [46] to analyze the stimulated Raman adiabatic passage (STIRAP) method [65]. To this end, we introduce the set of Gell-Mann matrices [51]

$$
\begin{array}{r}
\hat{\sigma}_{1}=\left(\begin{array}{ccc}
0 & 1 & 0 \\
1 & 0 & 0 \\
0 & 0 & 0
\end{array}\right), \quad \hat{\sigma}_{2}=\left(\begin{array}{ccc}
0 & -i & 0 \\
i & 0 & 0 \\
0 & 0 & 0
\end{array}\right), \\
\hat{\sigma}_{3}=\left(\begin{array}{ccc}
1 & 0 & 0 \\
0 & -1 & 0 \\
0 & 0 & 0
\end{array}\right), \quad \hat{\sigma}_{4}=\left(\begin{array}{ccc}
0 & 0 & 1 \\
0 & 0 & 0 \\
1 & 0 & 0
\end{array}\right), \\
\hat{\sigma}_{5}=\left(\begin{array}{ccc}
0 & 0 & -i \\
0 & 0 & 0 \\
i & 0 & 0
\end{array}\right), \quad \hat{\sigma}_{6}=\left(\begin{array}{ccc}
0 & 0 & 0 \\
0 & 0 & 1 \\
0 & 1 & 0
\end{array}\right), \\
\hat{\sigma}_{7}=\left(\begin{array}{ccc}
0 & 0 & 0 \\
0 & 0 & -i \\
0 & i & 0
\end{array}\right), \quad \hat{\sigma}_{8}=\frac{1}{\sqrt{3}}\left(\begin{array}{ccc}
1 & 0 & 0 \\
0 & 1 & 0 \\
0 & 0 & -2
\end{array}\right) .
\end{array}
$$



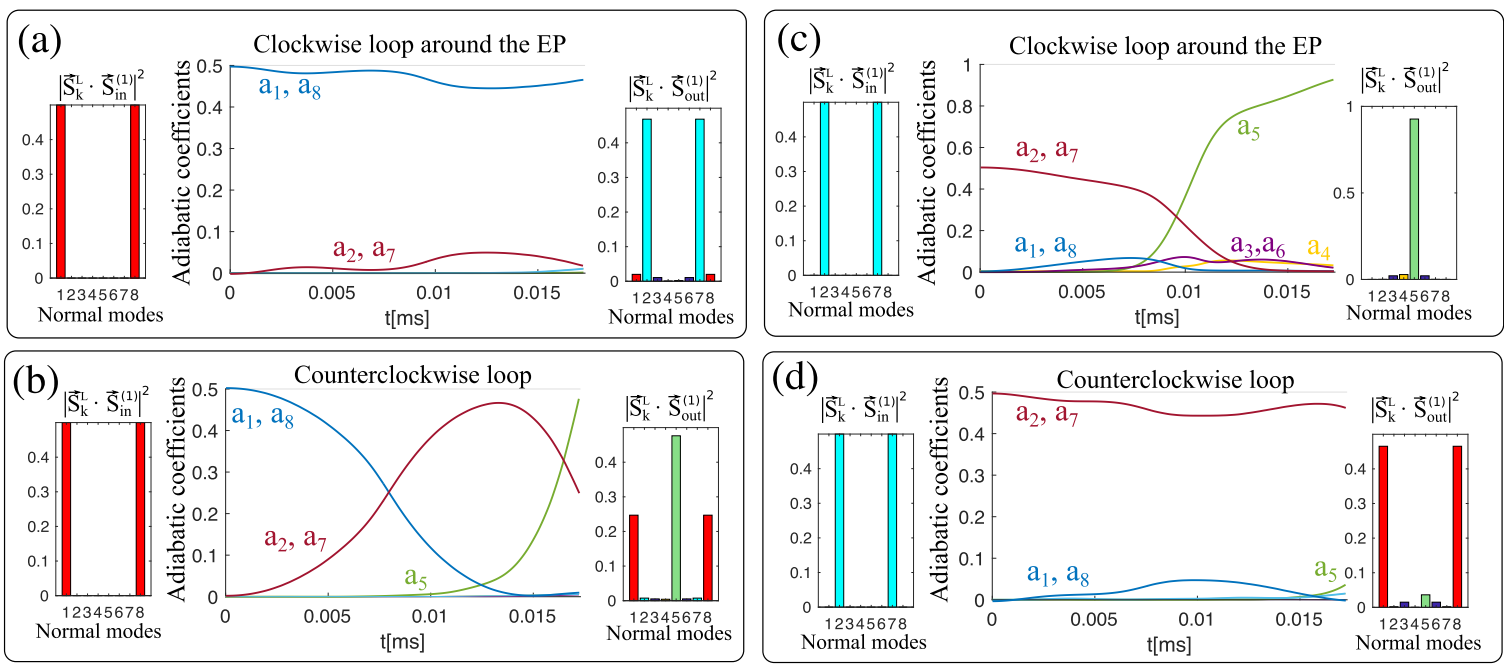

FIG. 5. Dynamically encircling an isolated EP. The middle panels (a)-(d) show the evolution of the adiabatic coefficients $\left|a_{i}(t)\right|^{2}=\mid \vec{S}_{i}^{L}(t)$. $\left.\vec{S}(t)\right|^{2}$ [Eq. (D2)] during the loop. The side panels in (a)-(d) show the normalized projections of the initial and final states onto the instantaneous states at the beginning of the loop, $\left|\vec{S}_{i}^{L}(0) \cdot \vec{S}_{\text {in/out }}^{(1,2)}\right|^{2}$. (a) When initialized in state $\vec{S}_{\text {in }}^{(1)}$ and evolved in a clockwise manner, the coefficients $a_{1}$ and $a_{8}$ are predominant throughout the evolution, which implies that the state evolves adiabatically, hence, the final state is the coalescing partner state $\vec{S}_{\text {in }}^{(2)}$. (b) When reversing the direction of the loop, the system does not evolve adiabatically and the final state is $\vec{S}_{5}^{R}$. (c), (d) Show the situation when the system is initialized in $\vec{S}_{\text {in }}^{(2)}$, where only a counterclockwise loop enables adiabatic mode swapping. The parameters are the same as in Fig. 3 from the main text.

We apply the Lindblad master equation [Eq. (3)] to each Gell-Mann operator $\left(\hat{\sigma}_{i}\right)$ and compute the expectation value, obtaining Eq. (5) from the main text, with

$$
\hat{M} \equiv\left(\begin{array}{cccccccc}
-\gamma_{11} & \Delta_{1} & 0 & 0 & 0 & 0 & \Omega_{2} & 0 \\
-\Delta_{1} & -\gamma_{22} & -2 \Omega_{1} & 0 & 0 & \Omega_{2} & 0 & 0 \\
0 & 2 \Omega_{1} & -\gamma_{33} & 0 & \Omega_{2} & 0 & 0 & \gamma_{38} \\
0 & 0 & 0 & -\gamma_{44} & \Delta_{2} & 0 & -\Omega_{1} & 0 \\
0 & 0 & -\Omega_{2} & -\Delta_{2} & -\gamma_{55} & \Omega_{1} & 0 & -\sqrt{3} \Omega_{2} \\
0 & -\Omega_{2} & 0 & 0 & -\Omega_{1} & -\gamma_{66} & -\left(\Delta_{1}-\Delta_{2}\right) & 0 \\
-\Omega_{2} & 0 & 0 & \Omega_{1} & 0 & \left(\Delta_{1}-\Delta_{2}\right) & -\gamma_{77} & 0 \\
0 & 0 & \gamma_{83} & 0 & \sqrt{3} \Omega_{2} & 0 & 0 & -\gamma_{88}
\end{array}\right),
$$

where we introduced the notation

$$
\begin{aligned}
\gamma_{11}=\gamma_{22} & \equiv\left(\frac{\gamma_{2}}{8}+\frac{\gamma_{1}+\kappa_{d}^{(1)}+\kappa_{u}^{(1)}+\kappa_{u}^{(2)}}{2}\right), \\
\gamma_{33} & \equiv \frac{\kappa_{u}^{(2)}}{2}+\kappa_{d}^{(1)}+\kappa_{u}^{(1)}, \\
\gamma_{38} & \equiv\left(\kappa_{d}^{(1)}-\kappa_{d}^{(2)}-\kappa_{u}^{(1)}-\frac{\kappa_{u}^{(2)}}{3}\right) \frac{1}{\sqrt{3}}, \\
\gamma_{44}=\gamma_{55} & \equiv \frac{\gamma_{1}}{8}+\frac{\gamma_{2}+\kappa_{u}^{(1)}+\kappa_{d}^{(2)}+\kappa_{u}^{(2)}}{2} \\
\gamma_{66}=\gamma_{77} & \equiv \frac{\gamma_{1}+\gamma_{2}}{8}+\frac{\kappa_{d}^{(1)}+\kappa_{d}^{(2)}}{2}, \\
\gamma_{83} & \equiv-\frac{\sqrt{3}}{2} \kappa_{u}^{(2)}, \\
\gamma_{88} & \equiv \frac{\kappa_{u}^{(2)}}{2}+\kappa_{d}^{(2)} .
\end{aligned}
$$

The steady-state vector is given by

$$
-\left(\hat{M}^{-1} \vec{S}_{\mathrm{eq}}\right)^{T}=\left(\begin{array}{llllllll}
0 & 0 & \frac{\Delta \kappa_{2}}{3}+\frac{2 \Delta \kappa_{1}}{3} & 0 & 0 & 0 & 0 & \frac{\Delta \kappa_{2}}{\sqrt{3}}
\end{array}\right)
$$

where we define the difference between upward and downward jumps as

$$
\Delta \kappa_{i} \equiv \kappa_{d}^{(i)}-\kappa_{u}^{(i)} .
$$

When the upward and downward rates are equal (i.e., in the high-temperature limit), the steady-state vector vanishes and the Gell-Mann vector will approach the origin at asymptotically large evolution times.

\section{APPENDIX C: HIGH-ORDER EPS IN A THREE-LEVEL SYSTEM}

In the main text, we show the existence of second- and third-order EPs in the ground-state manifold of the NV center. Here, we show that it is also possible to find highorder EPs in this system. For example, by searching the 
four-dimensional space spanned by $\Omega_{1,2}$ and $\Delta_{1,2}$ (see text), one can induce fourth- and fifth-order EPs, as shown in Fig. 4. More generally, the density matrix of an $N$-level system has $N^{2}-1$ real degrees of freedom. So, one could potentially find eighth-order EPs in this system, but that would require searching a higher-dimensional parameter space. In order to find a real degenerate eigenvalue of degree $M$, one needs $M-1$ real parameters [to satisfy $\left.\lambda_{1}(\vec{p})=\cdots=\lambda_{M}(\vec{p})\right]$. In order to find a complex degenerate eigenvalue of degree $M$, one needs $2(M-1)$ real parameters. We find high-order EPs by using the algorithm from Ref. [66], which exploits versal deformation theory for finding EPs of a given order.

\section{APPENDIX D: DYNAMICALLY ENCIRCLING AN ISOLATED EP}

In this Appendix, we provide additional information about the evolution of the system during the loop in parameter space when encircling the EP in both directions (i.e., clockwise and counterclockwise). We choose an elliptic path of the form

$$
\begin{aligned}
& \Delta_{1}(t)=\Delta_{\mathrm{EP}}+R_{\Delta} \cos (2 \pi t / T+\phi), \\
& \Omega_{1}(t)=\Omega_{\mathrm{EP}}+R_{\Omega} \sin (2 \pi t / T+\phi) .
\end{aligned}
$$

In Fig. 2, we use $\Delta_{\mathrm{EP}}=-80, \Omega_{\mathrm{EP}}=295, R_{\Delta}=100$, and $R_{\Omega}=30$ (all in $\mathrm{kHz}$ ), while in Fig. 3, the radii are $R_{\Delta}=260$ and $R_{\Omega}=125$. The phase is $\phi=0.39 \pi$ and the period is $T=15 / \gamma^{(1)}$.

When initialized in $\vec{S}_{\text {in }}^{(1)}$, the system evolves adiabatically only when the EP is encircled in a clockwise manner; the opposite is true for the second initial state, $\vec{S}_{\text {in }}^{(2)}$. At each moment along the evolution, the instantaneous normal modes form a complete basis of the Hilbert space. That is, the state vector at time $t$ can be written in the form

$$
\vec{S}(t)=\sum_{i=1}^{8} a_{i}(t) \vec{S}_{i}^{R}(t),
$$

where $a_{i}(t)$ are called "the adiabatic coefficients." We compute them be projecting the state vector $\vec{S}(t)$ onto the instantaneous basis states $\vec{S}_{i}^{L}(t)$. The system evolves adiabatically if it stays in the same instantaneous eigenstates throughout its evolution. It is important to emphasize that the instantaneous eigenstates themselves swap at the end of the loop; that is, if the system starts and ends with predominant coefficients $a_{1}$ and $a_{8}$, it means that the state swapped because $\vec{S}_{1}^{R}(t=T)=$ $\vec{S}_{2}^{R}(t=0)$ and $\vec{S}_{8}^{R}(t=T)=\vec{S}_{7}^{R}(t=0)$. Figure 5 shows the evolution of the adiabatic coefficients during the loop [middle panels in Figs. 5(a)- 5(d)] and the projections of the input and output states on the instantaneous eigenstates (i.e., the normal modes) at the beginning of the evolution. More details are given in the caption.
[1] T. Kato, Perturbation Theory for Linear Operators, Vol. 132 (Springer, Berlin, 2013).

[2] G. Strang, Linear Algebra and Its Applications (Thomson, Boston, 2006).

[3] L. N. Trefethen and M. Embree, Spectra and Pseudospectra: The Behavior of Nonnormal Matrices and Operators (Princeton University Press, Princeton, NJ, 2005).

[4] R. Uzdin, A. Mailybaev, and N. Moiseyev, On the observability and asymmetry of adiabatic state flips generated by exceptional points, J. Phys. A: Math. Theor. 44, 435302 (2011).

[5] M. V. Berry and R. Uzdin, Slow non-Hermitian cycling: Exact solutions and the Stokes phenomenon, J. Phys. A: Math. Theor. 44, 435303 (2011).

[6] T. J. Milburn, J. Doppler, C. A. Holmes, S. Portolan, S. Rotter, and P. Rabl, General description of quasiadiabatic dynamical phenomena near exceptional points, Phys. Rev. A 92, 052124 (2015).

[7] A. U. Hassan, G. L. Galmiche, G. Harari, P. LiKamWa, M. Khajavikhan, M. Segev, and D. N. Christodoulides, Chiral state conversion without encircling an exceptional point, Phys. Rev. A 96, 052129 (2017).

[8] A. U. Hassan, B. Zhen, M. Soljačić, M. Khajavikhan, and D. N. Christodoulides, Dynamically Encircling Exceptional Points: Exact Evolution and Polarization State Conversion, Phys. Rev. Lett. 118, 093002 (2017)

[9] J. Doppler, A. A. Mailybaev, J. Böhm, U. Kuhl, A. Girschik, F. Libisch, T. J. Milburn, P. Rabl, N. Moiseyev, and S. Rotter, Dynamically encircling an exceptional point for asymmetric mode switching, Nature (London) 537, 76 (2016).
[10] H. Xu, D. Mason, L. Jiang, and J. G. E. Harris, Topological energy transfer in an optomechanical system with exceptional points, Nature (London) 537, 80 (2016).

[11] J. W. Yoon, Y. Choi, C. Hahn, G. Kim, S. H. Song, K.-Y. Yang, J. Y. Lee, Y. Kim, C. S. Lee, J. K. Shin, H.-S. Lee, and P. Berini, Time-asymmetric loop around an exceptional point over the full optical communications band, Nature (London) 562, 86 (2018).

[12] X.-L. Zhang, S. Wang, B. Hou, and C. T. Chan, Dynamically Encircling Exceptional Points: In situ Control of Encircling Loops and the Role of the Starting Point, Phys. Rev. X 8, 021066 (2018).

[13] X.-L. Zhang, T. Jiang, H.-B. Sun, and C. T. Chan, Dynamically encircling an exceptional point in anti-PT-symmetric systems: Asymmetric mode switching for symmetry-broken states, arXiv: 1806.07649 .

[14] X.-L. Zhang and C. T. Chan, Hybrid exceptional point and its dynamical encircling in a two-state system, Phys. Rev. A 98, 033810 (2018).

[15] D. Chatzidimitriou and E. E. Kriezis, Optical switching through graphene-induced exceptional points, J. Opt. Soc. Am. B 35, 1525 (2018).

[16] P. R. Kaprálová-Žd'ánská and N. Moiseyev, Helium in chirped laser fields as a time-asymmetric atomic switch, J. Chem. Phys. 141, 014307 (2014).

[17] I. Gilary, A. A. Mailybaev, and N. Moiseyev, Timeasymmetric quantum-state-exchange mechanism, Phys. Rev. A 88, 010102(R) (2013).

[18] S. Ke, B. Wang, C. Qin, H. Long, K. Wang, and P. Lu, Exceptional points and asymmetric mode switching in plasmonic waveguides, J. Light. Technol. 34, 5258 (2016). 
[19] J. M. Taylor, P. Cappellaro, L. Childress, L. Jiang, D. Budker, P. R. Hemmer, A. Yacoby, R. Walsworth, and M. D. Lukin, High-sensitivity diamond magnetometer with nanoscale resolution, Nat. Phys. 4, 810 (2008).

[20] J. R. Maze, P. L. Stanwix, J. S. Hodges, S. Hong, J. M. Taylor, P. Cappellaro, L. Jiang, M. V. G. Dutt, E. Togan, A. S. Zibrov, A. Yacoby, R. Walsworth, and M. D. Lukin, Nanoscale magnetic sensing with an individual electronic spin in diamond, Nature (London) 455, 644 (2008).

[21] G. Balasubramanian, I. Y. Chan, R. Kolesov, M. Al-Hmoud, J. Tisler, C. Shin, C. Kim, A. Wojcik, P. R. Hemmer, A. Krueger, A. Leitenstorfer, R. Bratschitsch, F. Jelezko, and J Wrachtrup, Nanoscale imaging magnetometry with diamond spins under ambient conditions, Nature (London) 455, 648 (2008).

[22] F. Jelezko and J. Wrachtrup, Single defect centres in diamond: A review, Phys. Status Solidi A 203, 3207 (2006).

[23] M. Born and V. Fock, Beweis des adiabatensatzes, Z. Phys. 51, 165 (1928).

[24] T. Kato, On the adiabatic theorem of quantum mechanics, J. Phys. Soc. Jpn. 5, 435 (1950).

[25] S. Pancharatnam, Generalized theory of interference and its applications, in Proceedings of the National Academy of Sciences India, Section B, Vol. 44 (Springer, New York, 1956), pp. 398-417.

[26] H. C. Longuet-Higgins, U. Öpik, M. H. L. Pryce, and R. A. Sack, Studies of the Jahn-Teller effect. II. The dynamical problem, Proc. R. Soc. A 244, 1 (1958).

[27] M. V. Berry, Quantal phase factors accompanying adiabatic changes, Proc. R. Soc. A 392, 45 (1984).

[28] D. Xiao, M.-C. Chang, and Q. Niu, Berry phase effects on electronic properties, Rev. Mod. Phys. 82, 1959 (2010).

[29] C. Nayak, S. H. Simon, A. Stern, M. Freedman, and S. Das Sarma, Non-Abelian anyons and topological quantum computation, Rev. Mod. Phys. 80, 1083 (2008).

[30] L. Lu, J. D. Joannopoulos, and M. Soljačić, Topological photonics, Nat. Photonics 8, 821 (2014).

[31] C. A. Mead, The geometric phase in molecular systems, Rev. Mod. Phys. 64, 51 (1992).

[32] C. Dembowski, H.-D. Gräf, H. L. Harney, A. Heine, W. D. Heiss, H. Rehfeld, and A. Richter, Experimental Observation of the Topological Structure of Exceptional Points, Phys. Rev. Lett. 86, 787 (2001).

[33] G. Nenciu and G. Rasche, On the adiabatic theorem for nonselfadjoint Hamiltonians, J. Phys. A Math. Gen. 25, 5741 (1992).

[34] M. S. Sarandy and D. A. Lidar, Adiabatic approximation in open quantum systems, Phys. Rev. A 71, 012331 (2005).

[35] C. Miniatura, C. Sire, J. Baudon, and J. Bellissard, Geometrical phase factor for a non-Hermitian Hamiltonian, Europhys. Lett. 13, 199 (1990).

[36] M. W. Doherty, N. B. Manson, P. Delaney, F. Jelezko, J. Wrachtrup, and L. C. Hollenberg, The nitrogen-vacancy color center in diamond, Phys. Rep. 528, 1 (2013).

[37] Y. Chu and M. D. Lukin, Quantum Optics with NitrogenVacancy Centers in Diamond (Oxford University Press, Oxford, 2015).

[38] E. Togan, Y. Chu, A. Imamoglu, and M. D. Lukin, Laser cooling and real-time measurement of the nuclear spin environment of a solid-state qubit, Nature (London) 478, 497 (2011).

[39] Thiago P. Mayer Alegre, C. Santori, G. Medeiros-Ribeiro, and R. G. Beausoleil, Polarization-selective excitation of nitrogen vacancy centers in diamond, Phys. Rev. B 76, 165205 (2007).

[40] P. London, P. Balasubramanian, B. Naydenov, L. P. McGuinness, and F. Jelezko, Strong driving of a single spin using arbitrarily polarized fields, Phys. Rev. A 90, 012302 (2014).

[41] M. Mrózek, J. Mlynarczyk, D. S. Rudnicki, and W. Gawlik, Circularly polarized microwaves for magnetic resonance study in the GHz range: Application to nitrogen-vacancy in diamonds, Appl. Phys. Lett. 107, 013505 (2015).

[42] H. Haken, Laser Light Dynamics, Vol. 1 (North-Holland, Amsterdam, 1985).

[43] M. O. Scully and M. S. Zubairy, Quantum Optics (Cambridge University Press, Cambridge, 1999).

[44] H.-P. Breuer and F. Petruccione, The Theory of Open Quantum Systems (Oxford University Press, Oxford, 2002).

[45] M. Am-Shallem, R. Kosloff, and N. Moiseyev, Parameter estimation in atomic spectroscopy using exceptional points, Phys. Rev. A 93, 032116 (2016).

[46] T. Mathisen and J. Larson, Liouvillian of the open STIRAP problem, Entropy 20, 20 (2018).

[47] M. Am-Shallem, A. Levy, I. Schaefer, and R. Kosloff, Three approaches for representing Lindblad dynamics by a matrixvector notation, arXiv:1510.08634.

[48] M. S. Byrd and N. Khaneja, Characterization of the positivity of the density matrix in terms of the coherence vector representation, Phys. Rev. A 68, 062322 (2003).

[49] S. K. Goyal, B. N. Simon, R. Singh, and S. Simon, Geometry of the generalized Bloch sphere for qutrits, J. Phys. A: Math. Theor. 49, 165203 (2016).

[50] G. Kimura, The Bloch vector for N-level systems, Phys. Lett. A 314, 339 (2003).

[51] M. Gell-Mann, Symmetries of baryons and mesons, in Murray Gell-Mann: Selected Papers (World Scientific, Singapore, 2010), pp. 128-145.

[52] N. Moiseyev, Non-Hermitian Quantum Mechanics (Cambridge University Press, Cambridge, 2011), pp. 174-183.

[53] L. N. Trefethen and D. Bau III, Numerical Linear Algebra, Vol. 50 (SIAM, Philadelphia, 1997).

[54] M. Am-Shallem, R. Kosloff, and N. Moiseyev, Exceptional points for parameter estimation in open quantum systems: Analysis of the Bloch equations, New J. Phys. 17, 113036 (2015).

[55] D. Bohm, Quantum Theory (Dover, New York, 2012).

[56] I. Markovsky, Structured low-rank approximation and its applications, Automatica 44, 891 (2008).

[57] H. Abdi and L. J. Williams, Principal component analysis, Wiley Interdiscipl. Rev.: Comput. Stat. 2, 433 (2010).

[58] A. Bartana, R. Kosloff, and D. J. Tannor, Laser cooling of internal degrees of freedom: II, J. Chem. Phys. 106, 1435 (1997).

[59] H. Jirari and W. Pötz, Optimal coherent control of dissipative N-level systems, Phys. Rev. A 72, 013409 (2005).

[60] R. Roloff, M. Wenin, and W. Pötz, Optimal control for open quantum systems: Qubits and quantum gates, J. Comput. Theor. Nanosci. 6, 1837 (2009).

[61] Y. J. Yan, R. E. Gillilan, R. M. Whitnell, K. R. Wilson, and Shaul Mukamel, Optical control of molecular dynamics: Liouville-space theory, J. Phys. Chem. 97, 2320 (1993).

[62] C. M. Bender, S. Boettcher, and P. N. Meisinger, PT-symmetric quantum mechanics, J. Math. Phys. 40, 2201 (1999). 
[63] C. E. Rüter, K. G. Makris, R. El-Ganainy, D. N. Christodoulides, M. Segev, and D. Kip, Observation of parity-time symmetry in optics, Nat. Phys. 6, 192 (2010).

[64] Y. Wu, W. Liu, J. Geng, X. Song, X. Ye, C.-K. Duan, X. Rong, and J. Du, Observation of parity-time symmetry breaking in a single-spin system, Science 364, 878 (2019).
[65] N. V. Vitanov, T. Halfmann, B. W. Shore, and K. Bergmann, Laser-induced population transfer by adiabatic passage techniques, Annu. Rev. Phys. Chem. 52, 763 (2001).

[66] A. A. Mailybaev, Computation of multiple eigenvalues and generalized eigenvectors for matrices dependent on parameters, Numer. Linear Algebra Appl. 13, 419 (2006). 